\title{
Orbit Determination During Spacecraft Emergencies with Sparse Tracking Data - THEMIS and TDRS-3 Lessons Learned
}

\author{
Patrick J. Morinelli ${ }^{1}$ \\ Honeywell Technology Solutions Inc., Greenbelt, MD 20771 \\ Douglas T. Ward ${ }^{2}$ \\ a.i. solutions, Inc., Lanham, MD 20706 \\ Michael R. Blizzard ${ }^{3}$ \\ Honeywell Technology Solutions Inc., Greenbelt, MD 20771 \\ and \\ Chad R. Mendelsohn ${ }^{4}$ \\ NASA Goddard Spaceflight Center, Greenbelt, MD 20771
}

\begin{abstract}
This paper provides an overview of the lessons learned from the National Aeronautics and Space Administration (NASA) Goddard Space Flight Center's (GSFC) Flight Dynamics Facility's (FDF) support of the Time History of Events and Macroscale Interactions during Substorms (THEMIS) spacecraft emergency in February 2007, and the Tracking and Data Relay Satellite-3 (TDRS-3) spacecraft emergency in March 2006. A successful and timely recovery from both of these spacecraft emergencies depended on accurate knowledge of the orbit. Unfortunately, the combination of each spacecraft emergency with very little tracking data contributed to difficulties in estimating and predicting the orbit and delayed recovery efforts in both cases. In both the THEMIS and TDRS-3 spacecraft emergencies, numerous factors contributed to problems with obtaining nominal tracking data measurements. This paper details the various causative factors and challenges. This paper further enumerates lessons learned from FDF's recovery efforts involving the THEMIS and TDRS-3 spacecraft emergencies and scant tracking data, as well as recommendations for improvements and corrective actions. In addition, this paper describes the broad range of resources and complex navigation methods employed within the FDF for supporting critical navigation activities during all mission phases, including launch, early orbit, and on-orbit operations.
\end{abstract}

\footnotetext{
${ }^{1}$ Systems Engineer, NASA Goddard Space Flight Center Flight Dynamics Facility, NASA GSFC Mailstop 595.1, AIAA Member Grade MB

${ }^{2}$ Orbit Analyst, NASA Goddard Space Flight Center Flight Dynamics Facility, NASA GSFC Mailstop 595.1, AIAA Member Grade N/A

${ }^{3}$ Systems Engineer, NASA Goddard Space Flight Center Flight Dynamics Facility, NASA GSFC Mailstop 595.1, AIAA Member Grade N/A

${ }^{4}$ Orbit Analyst, NASA Goddard Space Flight Center Flight Dynamics Facility, NASA GSFC Mailstop 595.1, AIAA Member Grade N/A
} 


\section{Nomenclature}

$\begin{array}{ll}D S S & =\text { Deep Space Station } \\ U T C & =\text { Universal Time Coordinated }\end{array}$

\section{Introduction}

The Flight Dynamics Facility (FDF) was tasked to support Time History of Events and Macroscale Interactions during Substorms (THEMIS) in a limited capacity, providing backup orbit determination support for validation purposes for all five THEMIS probes during launch plus 30 days in coordination with the University of California Berkeley Flight Dynamics Center (UCB/FDC). However, various challenges starting on launch day, February 17, 2007, and a spacecraft emergency declared 30 hours after launch placed the FDF team in the role of providing the orbit solutions that enabled contact with each of the probes and the eventual termination of the spacecraft emergency. This paper details the challenges and various techniques used by the GSFC FDF team to successfully perform orbit determination for all five THEMIS probes during the early mission when there were sparse tracking measurements to support orbit determination and prediction.

The FDF provides routine orbit navigation services for all Tracking and Data Relay Satellites (TDRSs), including TDRS-3. On March 22, 2006, there was a spacecraft emergency which put TDRS-3 in Sun-pointing mode, causing thrusting and considerable orbit perturbation and tumbling. Contact with TDRS-3 was lost for 3 hours. During the recovery process, thrusters fired in an unknown configuration for nearly 2 hours and created an unexpected drift rate of 3 degrees per day. The 3-degree drift rate is unusually large, comparable to several repositioning burns, whereas a typical station keeping maneuver induces a drift rate change of $\sim 0.04$ degree per day. The TDRS controlling facility, White Sands Complex (WSC), had difficulty in characterizing the thruster firings. This paper details the tribulations and various orbit determination methods employed by the GSFC FDF team to recover the TDRS-3 spacecraft from the spacecraft emergency by successfully performing orbit determination with sparse tracking measurements.

\section{Spacecraft Emergency Summary}

\section{A. THEMIS Overview}

THEMIS is a National Aeronautics and Space Administration (NASA)-funded Medium Explorer mission (MIDEX) managed by the Explorers Program Office at GSFC in Greenbelt, Maryland. The THEMIS mission consists of five space probes, THEMIS A through THEMIS E, in elliptical low-inclination, high-Earth orbits. As the primary provider of flight dynamics services, the THEMIS Flight Dynamics Center (FDC), which is collocated with the Mission Operations Center (MOC) at the University of California at Berkeley (UCB), is responsible for performing orbit determination using the Goddard Trajectory Determination System (GTDS) through the entire mission and providing all acquisition data to the supporting ground stations. As backup to UCB/FDC, the FDF located at NASA GSFC was tasked to provide backup orbit determination using GTDS for the first 30 days of the mission and again during the Ascent Phase beginning in September 2007 for approximately 70 days. Therefore, the FDF team had a limited role in the navigation support for THEMIS. Orbit determination solutions and predictions are required for the support of onboard attitude processing, reduction of scientific measurements, and detailed maneuver planning, as well as generation of antenna acquisition data.

Constellation management is always a challenge, especially during the critical launch and early orbit mission phase. As stated, THEMIS is a constellation mission comprising five identical, yet separate spacecraft probes. All five probes were launched on and deployed from one Delta II launch vehicle. The Delta payload/probe carrier was designed such that THEMIS A was deployed in the velocity direction, whereas the remaining probes, THEMIS B through THEMIS E, were arranged around the carrier's circumference and deployed radially. There was no guidance telemetry at payload separation and the probe carrier was spinning. The Delta Acquisition Assistance Message (AAM) for THEMIS was generated after the 2nd stage "piloted" burn, using telemetry from the first two stages with the nominal 3rd stage performance added and propagated to payload separation. Unfortunately, the Delta AAM did not model residual thrusting or the spinning of the probe carrier. Thus there was no definitive separation state knowledge for any of the probes. The only distinction that could be made was that THEMIS A was to be released 3 seconds prior to THEMIS B through THEMIS E. Having only one spacecraft separation vector for all five probes introduced error into the critical launch day orbit determination process. However, any difference was estimated to be below the expected propagation error and any uncertainty of the last known state. 
An obvious flight dynamics challenge was managing all five probes simultaneously, particularly in their launch and early orbit phase. In addition, all five probes shared identical transponders with identical frequencies, thus increasing the potential for misidentified tracking data. Fortunately, these risks were never realized.

Tracking support for the five THEMIS spacecraft is provided by the NASA's Ground Network (GN) antennas, including the Wallops Flight Facility (WFF), Merritt Island (MIL), and Santiago, Chile (AGO) tracking stations. Although not a part of the NASA GN, both the Berkeley Ground Station (BGS) and the Hartebeesthoek Ground Station (HBK), in South Africa, also provide THEMIS tracking services, with BGS being the prime THEMIS Mission antenna. FDF was only tasked to validate HBK's ability to track and provide usable coherent Doppler tracking data. The ground station's tracking data had not been certified to any level of accuracy or quality prior to launch. UCB/FDC independently verified and accepted the HBK tracking data quality and accuracy.

\section{B. THEMIS Sequence of Events}

THEMIS was successfully launched on Friday, 2007-02-17 23:01:00 UTC. The Delta II ascent to orbit was nominal with THEMIS A separation occurring at 2007-02-18 00:14:14 UTC followed by THEMIS B through THEMIS E at 2007-02-18 00:14:17 UTC. The TDRS System (TDRSS) was scheduled to monitor the separation event, but no tracking data was provided since the TDRSS event was scheduled as a White Sands Complex Data Interface Service (WDISC) service, which provides telemetry and command data only. There were no problems noted during the TDRSS contact or the BGS contacts that immediately followed.

The Delta AAM state vector compared well with the pre-launch nominal; therefore, UCB/FDC decided not to update the network acquisition data. However, subsequent orbit solutions for all five probes indicated that the launch vehicle performance placed the THEMIS spacecraft $63.9 \%$ low on the achieved orbital period compared to the allowable launch vehicle dispersions. The launch vehicle dispersion allowances for the THEMIS orbit were $1999+/-$ 180 minutes for the orbital period, $435+/-6 \mathrm{~km}$ for perigee altitude, and $16+/-0.5$ degree for inclination. With such a large spread in orbital period permitted, the resultant orbit could produce extremely large instantaneous position errors compared to the mission nominal. Comparisons between the Delta AAM and pre-launch nominal could not identify the error in the Delta AAM vector, since it was a modeled propagation of a nominal 3rd stage performance. The unexpected launch vehicle performance translated into a 2-hour difference between the actual spacecraft period and the Delta AAM modeled separation state, with the actual period being 31.4 hours versus the 33.3-hour period modeled in the Delta AAM vector. See Table 1 for a comparison between the AAM separation state and that computed by FDF. This underperformance error in the Delta AAM state, which was used as the a priori state in the orbit determination process for all five probes, had an impact on critical early orbit determination and contributed to the THEMIS early mission difficulties.

Table 1. Comparison of the Delta AAM and the FDF Determined States

\begin{tabular}{|l|c|c|c|}
\hline \multicolumn{1}{|c|}{ Parameter } & AAM Value & FDF Value & Difference \\
\hline Epoch $(\mathrm{UTC})$ & $2008-02-1800: 14: 08.414$ & $2008-02-1800: 14: 08.414$ & 0 \\
\hline $\mathrm{X}(\mathrm{km})$ & 6484.8464 & 6485.26514941607 & -0.418749 \\
\hline $\mathrm{Y}(\mathrm{km})$ & -3998.9338 & -4007.03639711802 & 7.14302 \\
\hline $\mathrm{Z}(\mathrm{km})$ & -23.620889 & -22.9429770845846 & -0.677912 \\
\hline $\mathrm{Vx}(\mathrm{km} / \mathrm{s})$ & 7.3560188 & 7.34595828420958 & 0.0100605 \\
\hline $\mathrm{Vy}(\mathrm{km} / \mathrm{s})$ & 6.0284782 & 6.00830459333473 & 0.0201736 \\
\hline $\mathrm{Vz}(\mathrm{km} / \mathrm{s})$ & 2.5693697 & 2.57353917501606 & -0.00416948 \\
\hline $\begin{array}{l}\text { Osculating period } \\
(\mathrm{min})\end{array}$ & 1999.526 & 1884.305 & 115.221 \\
\hline $\begin{array}{l}\text { Osculating perigee } \\
\text { altitude }(\mathrm{km})\end{array}$ & 435.306 & 436.618 & -1.31436 \\
\hline $\begin{array}{l}\text { Osculating inclination } \\
\text { (deg) }\end{array}$ & 16.004 & 16.037 & -0.033 \\
\hline
\end{tabular}

Another contributing error was a spacecraft RF anomaly ${ }^{5}$ causing significantly weaker downlink signal strength than expected, which was aggravated by less than ideal antenna to ground orientations. Since all five probes shared identical RF designs, they all suffered from this RF communication anomaly. The onboard RF anomaly was further compounded by the apparent link margin problems observed with certain antennas tracking the THEMIS probes around apogee. The WFF 11-meter antenna experienced extreme difficulty acquiring the very weak THEMIS

${ }^{5}$ UCB THEMIS Status Briefing \& COMM Anomaly Tiger Team

American Institute of Aeronautics and Astronautics 082108 
downlink signal. The on-board spacecraft RF anomaly coupled with the poor spacecraft antenna geometry with respect to the supporting ground stations and the link margin problems all resulted in very little usable coherent Universal Tracking Data Format (UTDF) Doppler tracking data being collected during the critical initial orbit phase immediately following spacecraft separation. With the large errors in the propagated Delta AAM vector coupled with sparse coherent Doppler tracking data, initial orbit determination and estimation was difficult for THEMIS A, $\mathrm{B}$, and $\mathrm{E}$ and estimation of the THEMIS C and $\mathrm{D}$ was not possible. Without orbital estimation results, there were no antenna acquisition data updates available on launch day based on orbit determination.

The actual insertion orbit quickly diverged from that which was modeled in the pre-launch nominal acquisition data. In conjunction with the on-board RF anomaly, communication difficulties quickly escalated and eventually resulted in negative acquisitions, loss of communications with all probes, and ultimately in declaring a Spacecraft Emergency on 2007-02-19 04:30 UTC, only 30 hours after launch.

The majority of early mission passes on the THEMIS probes did not result in a successful acquisition of signal. The stations often then shifted to manual track, sweeping the antennas for a "hit" on the spacecraft. Tracking data from these contacts had to be used very judiciously, as all the resultant measurements were not good except for a couple, as the antenna was in effect on program track, with no RF from the spacecraft. In order to use this data, it was necessary for orbit determination analysts to talk to the ground station to determine if the antenna operator got any "hit" or contacts on the spacecraft during the sweep.

\section{Detailed THEMIS Tracking Challenges}

Although GN UTDF Doppler data was the only expected tracking data type prior to launch, angle tracking data from various supporting GN sites was absolutely critical during the first few days after launch for orbit determination and Spacecraft Emergency recovery. Of the certified THEMIS GN sites, only Wallops (WFF), Merritt Island (MIL), and Santiago (AGO) have the capability to provide angle tracking measurements. With the RF and link margin issues, angle tracking data came into FDF flagged invalid because there was a low signal to noise ratio, which prevented the validity trigger in the antenna system, hence all of the tracking data was marked invalid from launch forward for THEMIS. FDF evaluated the invalid angle data and determined that, although the measurements were invalid, the angle tracking was usable. In order to use the invalid angle tracking data in the orbit solutions, FDF had to manually configure the orbit system to override the validity flag, which was crucial in getting the solutions that enabled communication with the THEMIS probes.

Over the first few days after THEMIS launch, using the initial Wallops pass, with data flagged as invalid from THEMIS A, FDF was able to generate and provide the best initial post-launch orbit solution that was used to update the station acquisition data, re-establishing communication with all five probes and terminating the Spacecraft Emergency. This solution was built from a couple of manual track hits on THEMIS A, as well as the first contact right after separation from BGS. This solution was crucial to recovery of all the mission orbits due to the quickly evolving Spacecraft Emergency. It was only by bootstrapping this initial solution for THEMIS A, using the Wallops angle data, that good orbit solutions were achievable for THEMIS B through THEMIS E.

In an attempt to improve communication with the probes using antennas with greater link margins, the HBK facility began their support using uplinks and downlinks from the 10-meter antenna. Due to the troubled downlinks, the station attempted multiple configurations, eventually settling on a dual configuration consisting of a 6-meter transmit antenna and a 12-meter receive antenna. However, the tracking data from this HBK dual configuration was not properly labeled, because the UTDF tracking data from these events still used the antenna identifiers of the 10meter antenna, as this was the expected nominal antenna validated for THEMIS support by UCB/FDC. In order to correctly process and use the HBK 6-meter/12-meter antenna tracking data, FDF had to manipulate the tracking data and overwrite the transmit and receive antenna identification codes. The 6-meter/12-meter antenna configuration was a new antenna for FDF and not included in the station files used by the FDF or UCB/FDC orbit ground systems. In order to correctly process and use the HBK 6-meter/12-meter tracking data, FDF had to manually configure the orbit determination system to correctly recognize the tracking station and use the correct station geodetic information. FDF reset this data to be the proper stations by overwriting the transmit and receive sites on its Oracle SQL database used to store the raw data. Since the HBK 6-meter/12-meter was a previously unused antenna configuration, FDF first had to quickly research and analyze the geodetics for this antenna to ensure proper performance while under this Spacecraft Emergency, a process that is generally performed with multi-station tracking with known references for comparisons. This process was repeatedly necessary for the constellation of THEMIS spacecraft through the early mission support. Existing lines of communication between FDF and various elements of the tracking networks were crucial to verifying the HBK configurations used for each pass. Unfortunately, UCB/FDC had no convenient means of properly overwriting and processing this tracking data. FDF extracted the reprocessed data from its Oracle database, converted it to 60-byte sequential GTDS input tracking data 
files $^{6}$, and sent this data to UCB/FDC. FDF provided consultation on how to manually configure orbit systems to use correct station geodetic information.

Orbit determination was originally planned to be based exclusively on two-way NASA GN UTDF Doppler tracking data. Spacecraft RF link margin issues and problems with antenna patterns during the early orbit mission phase led the FDF team to recommend additional support from the NASA Deep Space Network (DSN) after the THEMIS Spacecraft Emergency was issued. Therefore, although the GN antennas were the primary source of tracking data for THEMIS, DSN tracking data supplemented the GN data and was critical to early orbit estimation. Since the DSN was not configured to support THEMIS, the DSN sent all the THEMIS tracking data identified as TDRS-10 or as ChipSat, satellites they had supported before. This required FDF to perform data overrides for all of the DSN tracking data, as well; again, a laborious and intensive project while supporting five probes. Since UCB/FDC was not configured for DSN support, no network interface existed between UCB/FDC and the DSN. FDF generated and delivered to the DSN acquisition data to enable the antenna support for UCB/FDC. In addition, UCB/FDC could not command THEMIS nor receive DSN telemetry or tracking data. There were a number of issues related to the DSN not being configured for supporting THEMIS that impacted FDF support. The DSN was able to track only in a three-way mode when another one of the THEMIS certified GN sites was tracking simultaneously. In order to properly process and use the DSN and simultaneous GN tracking data, FDF had to manipulate the data to add in three-way overrides to the appropriate tracking measurements. Since in the majority of cases the data had already been accumulated, the data had to be replayed to reset the 3-way overrides to the data, or the data had to be directly manipulated to be the correct configuration. Again, this was laborious for five spacecraft throughout the support. Since GN Doppler UTDF tracking data was the only expected data type that UCB/FDC expected to receive prior to launch, the UCB/FDC orbit system was only configured to support this data type. The DSN provided valuable angle data, as well as Jet Propulsion Laboratory (JPL) TRK-2-34 formatted Doppler data types during THEMIS support. In order for UCB/FDC to be able to process and use the DSN tracking data correctly, FDF provided a 60-byte sequential input tracking data file to UCB/FDC and consultation on how to set up and use this tracking data type in GTDS

\section{THEMIS Lessons Learned}

For missions that have large possible launch dispersions, even a slight difference from nominal could produce situations that will rapidly degrade into contingency situations. The best posture for these situations is to be prepared before launch to work with the launch dispersion cases quickly. Further, using launch vehicle inertial guidance data can be risky, especially when the final stages are only modeled. It is better to ensure that the launch vehicle data is available from all stages, a process that can be a mission criterion for the launch planning. It is better yet to ensure that the launch vehicle is tracked by ground antennas with tracking data to enable an independent verification of the separation state. This technique is often used for FDF-supported missions. Used in combination with a spectrum of possible dispersion data, the data will quickly yield the correct launch information for the new missions.

The prime consideration during an early orbit period is to determine the newly launched mission's orbit as quickly as possible, to prevent the outcome of poor acquisition and communications, as happened in this complicated support for the THEMIS mission. The THEMIS situation was also greatly exacerbated by the simultaneous support of five spacecraft, which also constrained the possible tracking contacts dedicated to any of the spacecraft.

THEMIS has been successfully recovered from its Spacecraft Emergency. FDF continued to support UCB/FDC through launch plus 60 days, an extension from the original launch plus 30 day support plan. After the post-launch 60-day support period, FDF support subsided to a proficiency level of only one orbit solution per probe per month.

\section{TDRS-3 Overview}

The NASA TDRSS enables communication with and tracking of Earth-orbiting spacecraft. The system is composed of nine spacecraft in low-inclination geosynchronous orbits positioned in assigned longitudinal slots around the Earth and the ground systems used to track and control them. A TDRS is controlled through the WSC located in White Sands, New Mexico, under the direction of the Space Network (SN) Project of Code 452 at NASA's GSFC. The FDF at GSFC is responsible for TDRS orbit determination and prediction.

\footnotetext{
${ }^{6}$ The generalized 60-byte record format serves as a uniform medium for passing metric tracking data with standardized units as input to GTDS. National Aeronautics and Space Administration (NASA), Goddard Space Flight Center (GSFC), 60-Byte Data Format Definition
} 
Nominal tracking data support for TDRS-3 includes S-band Bilateration Ranging Transponder System (BRTS) tracking from Alice Springs, Australia, every 4 hours and K-band Tracking Telemetry and Command (TT\&C) data from Guam (GRGT) every hour.

\section{TDRS-3 Sequence of Events}

On March 22, 2006, TDRS-3 had an Emergency Time Out (ETO) resulting in an attitude divergence leading to a protracted period of loss of service. The anomaly included significant thrusting for more than 2 hours until near 0300 UTC, which accounted for nearly $90 \%$ of the total thrusting during the ETO. Minor thrusting continued for another 10 hours until approximately 1300 UTC and accounted for the remaining $10 \%$ of the total thrusting experienced during the ETO. The spacecraft was then drifting westward at a rate of 3 degrees per day. The FDF found that the orbit change from the thrusting was approximately $4,380 \mathrm{~km}$ in orbital position after 32 hours. Table 2 shows the order of events adapted from the timeline as reported by the FDF.

Table 2. TDRS-3 2006 Anomaly

\begin{tabular}{|l|l|}
\hline Date & Event \\
\hline $3 / 22$ & Loss of attitude control and loss of Earth reference. \\
\hline $3 / 22$ & End of 90 percent of thrusting. \\
\hline $3 / 22$ & $\begin{array}{l}\text { End of last } 10 \text { percent of thrusting. Tracking data taken during this period were } \\
\text { unusable due to thrusting. }\end{array}$ \\
\hline $3 / 22$ & TDRS-3 returned to normal mode, S-band TT\&C tracking only. \\
\hline $3 / 22$ & FDF provided solution based on pre-maneuver data. \\
\hline $3 / 22$ & First pass of DSS-46 data received. \\
\hline $3 / 23$ & Third and last pass of DSS-46 data received. \\
\hline $3 / 23$ & FDF reported being unable to generate a usable solution from the data. \\
\hline $3 / 23$ & $\begin{array}{l}\text { The first post-tumble United States Strategic Command (USSTRATCOM) solution } \\
\text { was received. }\end{array}$ \\
\hline $3 / 23$ & $\begin{array}{l}\text { FDF obtained a converged solution from three Canberra passes over a 7-hour arc } \\
\text { using the USSTRATCOM vector as initial conditions. }\end{array}$ \\
\hline $3 / 23$ & FDF delivered a converged solution. \\
\hline $3 / 24$ & Maneuver to stop drift. \\
\hline $3 / 24$ & Maneuver to return the spacecraft to its box (to start drift back). \\
\hline $3 / 24$ & Resumption of BRTS events. \\
\hline $3 / 28$ & Maneuver to slow drift. \\
\hline $3 / 28$ & Maneuver to stop drift and stay in the box. \\
\hline $3 / 28$ & TDRS-3 emergency terminated. \\
\hline
\end{tabular}

\section{Detailed TDRS-3 Tracking Challenges}

To avoid interference with other spacecraft, K-band tracking was prohibited, which barred S-band BRTS tracking data and GRGT K-band TT\&C data. (K-band space-to-ground service is required to achieve TDRS-toUser tracking in either S- or K-band.) Being only able to program track, Guam was unable to provide any usable angle data for orbit determination. Further eliminating Guam tracking data were very large and changing range biases. Guam had at least four groups of range data biased by integrals of $\sim 37.5 \mathrm{~km}$, ranging from between -412 and $-640 \mathrm{~km}$.

As the contingency tracking station for TDRS-3, the Canberra, Australia Deep Space Station, DSS-46, assisted in the recovery efforts. Given the low elevations for observing TDRS-3 from Canberra, DSS-46 had difficulty maintaining lock. Further exasperating the situation was competition from the concurrent Space Technology 5 (ST5) mission's launch and early orbit support for both DSN and FDF staff and resources.

The data received from the Canberra site were the only good data received by the time the first orbit update was attempted at 01:00 UTC on March 23. However, the data covered only slightly more than a quarter of the orbit, not enough for a solution with data from a single station and with poorly known a priori elements. In response to a large unplanned orbit change, this initial orbit solution was poor with very little tracking data, much less than normal operations, and also had inadequate tracking geometry.

It was not until USSTRATCOM provided a better initial state vector that the FDF was successful in processing the tracking data and updating the TDRS-3 orbit at approximately 2100 UTC on March 23. This initial orbit solution 
was based on selective tracking data. DSS-46 Doppler data and Guam range data were excluded whereas angle data from both Guam and DSS-46 were included with DSS-46 range data.

When it was recognized that the Guam TT\&C data had large biases in several time periods with different multiples of $37.5 \mathrm{~km}$, attempts were made to remove the biases of 400 to $600 \mathrm{~km}$. Three segments of range data were isolated with a segment-specific bias applied. This is not a built-in feature of GTDS and is described in a similar use in Reference 1. This effort had progressed sufficiently to corroborate the orbit solution obtained from using the DSS-46 range, the DSS-46 and Guam angle data, and the USSTRATCOM solution vector as input. In later TDRS-3 recovery support, this segmented-range bias effort matured sufficiently so that biased range data from Guam was corrected enough to be used in an orbit solution.

There was a $466 \mathrm{~km}$ difference between the WSC predicted reconstructed vector versus the USSTRATCOM post-maneuver vector, with $50 \mathrm{~km}$ of the error being in the cross-track component. There was a 4,324 km difference between the WSC predicted reconstructed vector and the TDRS-3 FDF operational no-burn ephemeris. This means there was a 4,324 km change in the orbit over 2 days, which reflects a drift of 3 degrees per day. It was later learned that the WSC reconstructed vector had a large error in the roll direction.

\section{TDRS-3 Lessons Learned}

TDRS tracking of a BRTS transponder requires a K-band space-to-ground link (SGL). Once a TDRS transitions to S-band during an emergency, it no longer maintains BRTS lock. When significant out-of-plane thrusting has occurred and BRTS events are not promptly resumed, additional tracking support will be required. Over a short arc with no maneuver modeling, the FDF may be unable to generate a reliable orbit solution based on TT\&C data from one station after a large perturbation, unless additional steps are taken. The segmented-range bias modeling was able to salvage data that was 400 to $600 \mathrm{~km}$ off. Another step that could be taken is using range residual graphs as feedback to tune Delta-Velocity modeling after a preburn input vector, including 2- or 3-dimensional burn modeling, including out of plane burning. The burn modeling could be segmented into multiple burn times, if appropriate. Various orbit determination solution options and features were attempted throughout the recovery process. However, none of these produced a usable solution until the USSTRATCOM Two-Line Element (TLE) was available.

\section{Conclusion}

During a contingency situation, the expectation is that tracking would be performed from any asset available to support. FDF can attempt orbit determination when sufficient tracking data is available. However, there may be some limitations in the data that would reduce their effectiveness for orbit support, such as short data arcs, data dropouts from spacecraft spin, or even spacecraft related anomalies, or a lack of calibration of the tracking station equipment. If difficulty continues in generating an orbit solution, coordination and assistance from USSTRATCOM or additional measures may be sought.

Contingency planning and extensive resources are strengths of the FDF. Drawing on experiences of launching and supporting hundreds of spacecraft missions, the FDF has built tools that permit data manipulation for contingencies and have the capability of using more than 23 data types to expand the possibilities of supporting new missions and aid in the recovery of spacecraft during spacecraft emergencies. FDF personnel analytical capabilities and experience also supply needed assistance during these endeavors.

One of the primary objectives that FDF personnel use in planning critical orbit support is to build into the scenario the capability to use as many data types as possible due to possible contingencies with any one or more data types. In the past, FDF has put together critical orbit support schedules that included as many as five different data types. In one instance as many as three of these data types were not available for various reasons. Hence it is better to be prepared for such contingencies during these critical few hours. The fewer data types during critical support, the greater the risk.

Recognition that greater resources and planning will prevent far greater expenditures to recover a mission from Spacecraft Emergency or other anomalous situations will result in long-term savings and may prevent an irrecoverable loss.

\section{References}

${ }^{1}$ Dang, K., Ward, D., Slojkowski, S., Dunham, J., and Blizzard, M., “Tracking And Data Relay Satellite (TDRS) Orbit Determination Using Chain-Dependent Range Biases," 2005 Flight Mechanics Symposium, Greenbelt, Maryland, October 2005, NASA/CP-2005-212789, Session 4, Paper No. 4. 